\title{
Analysis of Influence and Contribution on Distribution System under Widespread PVs and EVs
}

\author{
Shoji Kawasaki and Shunsuke Fukami \\ Department of Electronics and Bioinformatics, School of Science and Technology, Meiji University, Kanagawa 214-8571, Japan
}

\begin{abstract}
In recent years, against a background of an environmental problem and resource problem, the introduction of RES (renewable energy source) such as wind power generation and PV (photovoltaic generation), EV (electric vehicle), and PHEV (Plug-in hybrid electric vehicle) has been expanding. However, various problems have an ongoing discussion. When the production of electricity by RESs exceeds the power consumption, it is possible to cause a steep variation of point voltage and a deviation from a proper voltage range in a distribution system to which RESs are interconnected. When EVs and PHEVs have spread to the distribution system, a new peak power-demand and a steep voltage drop might occur in the midnight charging time zone in case the electricity charges are low. In this paper, the authors analyze the effects on the distribution system under widespread PVs, EVs, and PHEVs. In addition, the authors propose an improvement plan and analyze about the influence and contribution.
\end{abstract}

Key words: Photovoltaic generation, electric vehicle, reverse power flow, battery charge, plug-in hybrid electric vehicle.

\section{Introduction}

In recent years, the number of RES such as PV and wind power generation system that interconnects to a distribution system has been increasing to reduce the influence on the environment in Japan. And the number of an automobile powered by electricity such as EV and PHEV has been increasing too. The output of $\mathrm{PV}$ is influenced by change of weather conditions and causes a rapid fluctuation. When the production of electricity by RESs exceeds the power consumption, it is possible to cause a reverse power flow, steep change of point voltage, deviation from a proper voltage range, etc. In addition, when EVs and PHEVs have spread to the distribution system, a new power-demand and steep voltage drop might occur in the midnight charging time zone in case the electricity charges are low in Japan.

In this study, firstly, the authors build a detailed analysis model of general distribution system in Japan, and analyze the effects on the distribution system

Corresponding author: Shoji Kawasaki, senior assistant professor, research fields: optimization control of distribution network and electric power quality improvement of distribution network. introduced a demand response which changes the electric power usage according to the situation if the electric power supply and demand under widespread of PVs, EVs, and PHEVs. Next, the authors propose a new charging schedule for EVs and PHEVs, and analyze the influence and contribution on the distribution system introduced the proposed schedule under a variety of conditions. Finally, the authors summarize the results of analyses mentioned above as an index, and indicate it as a part of electric power system planning.

\section{Analyses Condition}

\subsection{Distribution System Model}

Fig. 1 shows the used analytical model. The model was assumed the general three-phase (phase $\mathrm{U}$, phase $\mathrm{V}$ and phase $\mathrm{W}$ ) unbalanced distribution system of residential area in Japan. The electric power is supplied to loads through a distribution substation from a higher system like Fig. 1. The line length between the nodes is $0.5 \mathrm{~km}$ (total line length is 3.5 $\mathrm{km}$ ), and each line is assumed the aluminum wire (AL-OE120). Also, this feeder is composed of 9 loads, 


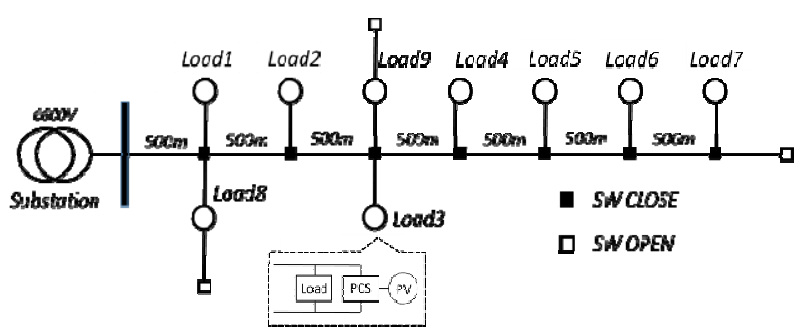

Fig. 1 Distribution system model of residential area.

9 pole transformers, and 9 PV systems. The used loads assume $100 \mathrm{~V}$ or $200 \mathrm{~V}$ residential load are connected to the secondary distribution system thorough the pole transformers.

\subsection{Power-Demand Curve}

Fig. 2 shows the used power-demand curve of residential area [1]. In this study, the authors use the 2 patterns power-demand curve. One is a curve of heavy load period in case electric demand increases, and the other is a curve of light load period in case electric demand decreases. The total load capacity of feeder in heavy load period and in light load period is $2.8 \mathrm{MW}$ and 1.6 MW, respectively. The number of houses connected to the feeder is 2,300. The voltage imbalance factor of feeder in heavy load period and in light load period is $1.31 \%$ and $1.21 \%$, respectively.

\subsection{Model of PVS}

Fig. 3 shows used PV output of one house of the clear day. The PV output approaches the peak at 12 a.m., and the peak output is $3 \mathrm{~kW}$. In this study, the power factor for the interconnection of PV system is 1 . And the influence and contribution on distribution system are analyzed by changing the penetration rate of PVs.

\subsection{Distribution Ratio of PVS}

When the number of PVs spread to the distribution system is concentrating certain area, it is considered that it affects the power flow of distribution system greatly. Therefore, in this study, the authors divided the distribution system into three areas (area A, area B, and area C) as shown in Fig. 4, and verified the power

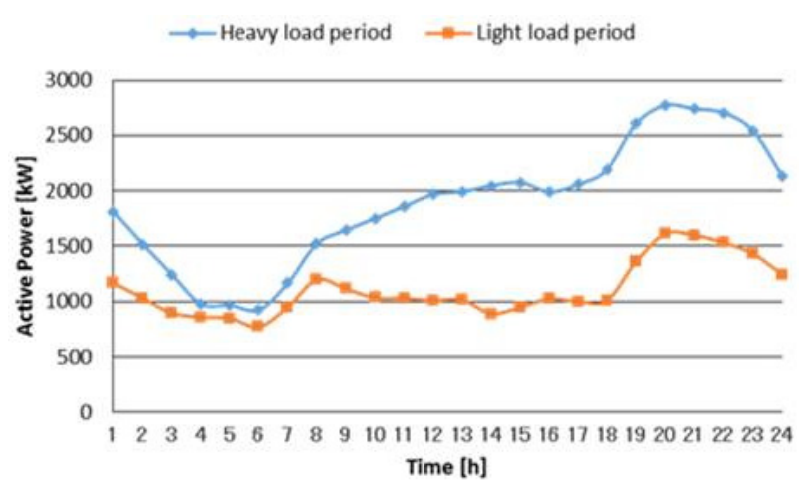

Fig. 2 Power-demand of distribution system.

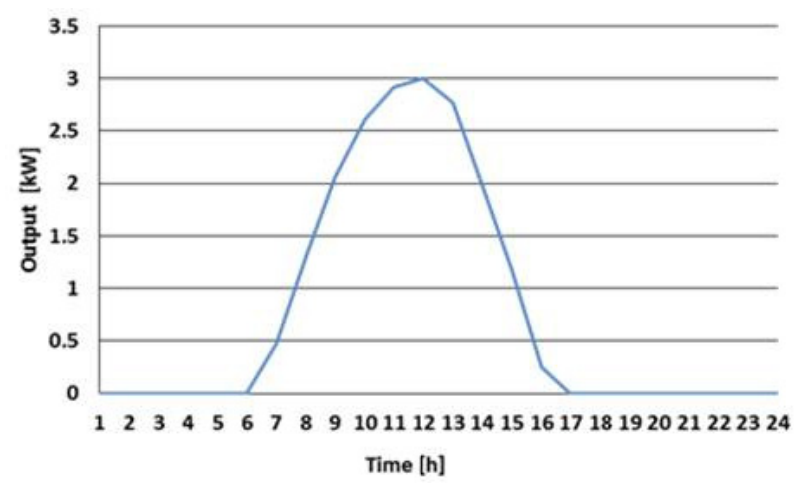

Fig. 3 PV output per house.

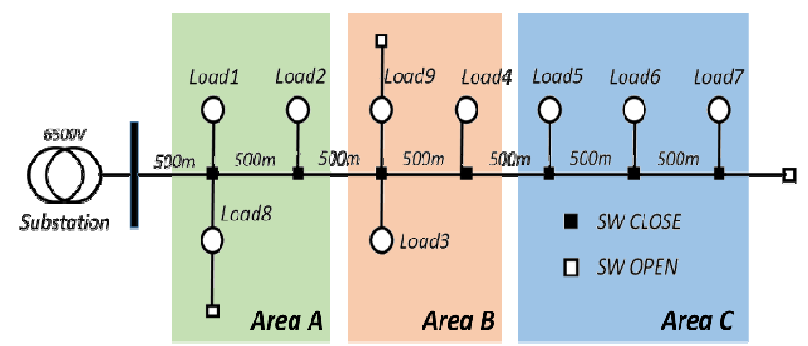

Fig. 4 Divided areas in distribution system.

Table 1 Distribution ratio of PVs.

\begin{tabular}{|c|ccccc|}
\hline & Area A & & Area B & & Area C \\
\hline Case 1 & 1 & $:$ & 1 & $:$ & 1 \\
Case 2 & 2 & $:$ & 1 & $:$ & 1 \\
Case 3 & 1 & $:$ & 2 & $:$ & 1 \\
Case 4 & 1 & $:$ & 1 & $:$ & 2 \\
\hline
\end{tabular}

flow under the four cases of distribution ratio of PVs as shown in Table 1. In this study, the power flow is analyzed by the phase differences between the loads.

\subsection{Simulation Condition of EVs and PHEVs}

Table 2 shows the details of simulation condition of EVs and PHEVs [2]. The introduction rate of EVs and 
Table 2 Simulation condition of EV and PHEV.

\begin{tabular}{lcl}
\hline \multirow{2}{*}{ Battery Capacity } & EV & $24 \mathrm{kWh}$ \\
& PHEV & $4.4 \mathrm{kWh}$ \\
\hline \multirow{2}{*}{ Charging method } & EV & $3 \mathrm{~kW}$ (constant power charge) \\
& PHEV & $2 \mathrm{~kW}$ (constant power charge) \\
\hline
\end{tabular}

Table 3 Number of EVs and PHEVs at each load.

\begin{tabular}{|c|c|c|c|c|}
\hline & & The number of houses & The number of EVs & The number of PHEVs \\
\hline \multirow{7}{*}{\begin{tabular}{c} 
Load \\
\cline { 2 - 5 }
\end{tabular}} & 1 & 207 & 47 & 15 \\
\cline { 2 - 5 } & 2 & 299 & 68 & 22 \\
\cline { 2 - 5 } & 3 & 230 & 52 & 17 \\
\cline { 2 - 5 } & 4 & 253 & 57 & 19 \\
\cline { 2 - 5 } & 5 & 230 & 52 & 17 \\
\cline { 2 - 5 } & 6 & 276 & 62 & 20 \\
\cline { 2 - 5 } & 7 & 253 & 57 & 19 \\
\cline { 2 - 5 } & 8 & 253 & 57 & 22 \\
\cline { 2 - 5 } & 9 & 299 & 68 & 170 \\
\hline
\end{tabular}

PHEVs is $30 \%$, which is the spread target value of Japanese government by 2030 [3]. And the ratio of EVs and PHEVs is 3 to 1 [4]. Table 3 shows the number of houses, EVs and PHEVs which each load has.

In this study, all EVs and PHEVs charge batteries by a normal charger, so only electric vehicles stay home can start charging. The authors set whether electric vehicles stay home or not Eq. (1).

$$
H_{\text {car }}=\left\{1-\left(1-H_{\text {home }}\right) \times A\right\} \times 100
$$

where, $H_{c a r}[\%]$ is hourly rate of electric vehicles stay home, $H_{\text {home }}$ is hourly rate of people stay home, and $A$ is rate of car users occupied to main means of transportation.

\subsection{Charging Schedule for EVs and PHEVS}

In this study, EVs and PHEVs charge the battery by the following two kinds of charging schedules, which are as follows:

Charging schedule 1: "Night simultaneously charging schedule";

Charging schedule 2: "Split charging schedule".

Charging schedule 1 based on the assumption that all EVs and PHEVs stay home at 11 p.m. in case the electricity charges are low in Japan, and the charge is started from this time.

Fig. 5 shows the rate of people who stay home and rate of car users at 11 p.m. $[5,6]$. As shown in Fig. 5,
$H_{\text {home }}$ at 11 p.m. is $87 \%, A$ at 11 p.m. is $45.7 \%$. Hence, $H_{c a r}$ which calculated by Eq. (1) is $94.1 \%$, and this percentage of EVs and PHEVs start charging at 11 p.m.

In charging schedule 2, EVs and PHEVs stay home at 12 a.m. when PV output approaches the peak, and the charge is started from this time. And EVs and PHEVs charge surplus power produced by PVs. The others start charging at 11 p.m.

Fig. 6 shows the rate of people stay home and rate of car users at 12 a.m. [5, 6]. As shown in Fig. 6, $H_{\text {home }}$ at 12 a.m. is $28 \%, A$ at 12 a.m. is $45.7 \%$. Hence, $H_{c a r}$ which calculated by Eq. (1) is $67.1 \%$, and this percentage of EVs and PHEVs start charging at 12 a.m. and the others start charging at 11 p.m. by the same schedule as 1. All EVs and PHEVs do full charge.

\subsection{Configuration of Remaining Battery}

Electric vehicle owners have various life patterns, and there are differences in the mileage on the EVs and PHEVs. Therefore, the remaining battery when EVs and PHEVs start charging should be set distinctively. Figs. 7 and 8 shows percentage of EVs and PHEVs for the remaining battery when start charging [7].

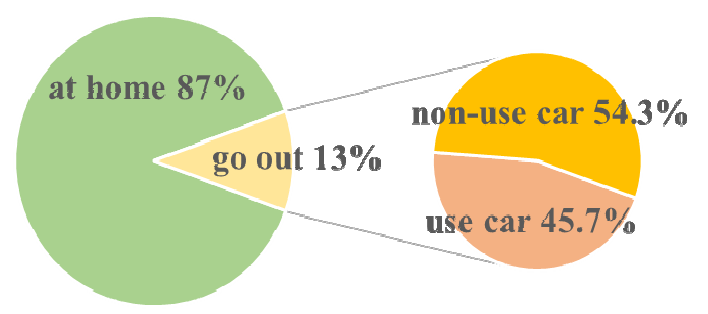

Fig. 5 Rate of people at home and of car users (at 11 p.m.).

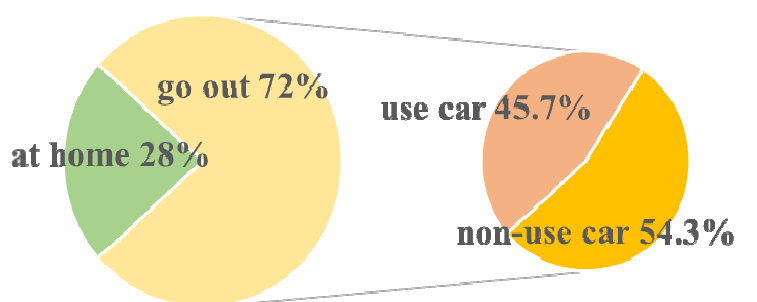

Fig. 6 Rate of people at home and of car users (at 12 a.m.). 


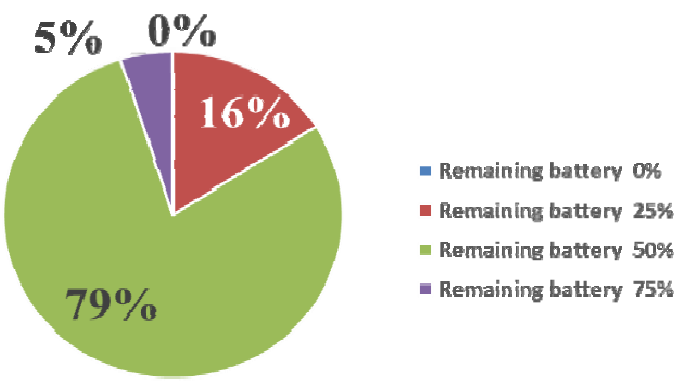

Fig. 7 Percentage of EVs for remaining battery when start charging.

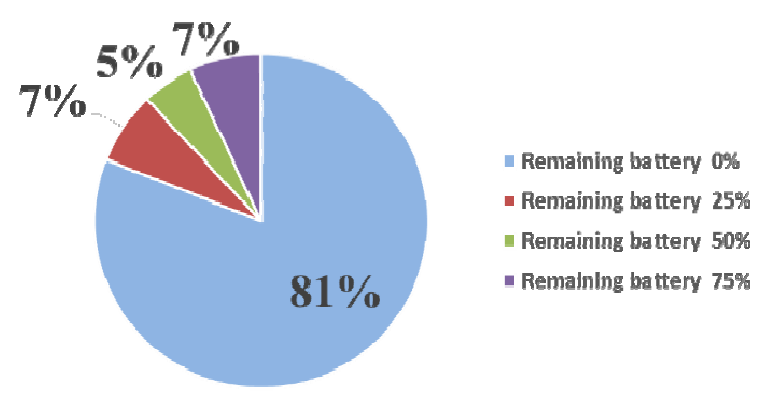

Fig. 8 Percentage of PHEVs for remaining battery when start charging.

\section{Verification of Influences by EVs and PHEVs Charging}

Figs. 9 and 10 show the power-demand curve of residential area of heavy load period and light load period by each charging schedule. In Figs. 9 and 10, the power-demand rises rapidly at $11 \mathrm{p} . \mathrm{m}$. by the night simultaneously charging schedule. The rise of power-demand is suppressed by the split charging schedule compared with the night simultaneously charging schedule. Instead, the power-demand increases in the daytime.

Fig. 11 shows the power-demand at 11 p.m. and Figs. 12-14 show the line voltage at 11 p.m. by each charging schedule. In Fig. 11, the power-demand by the night simultaneously charging schedule is about $1,500 \mathrm{~kW}$ higher than it by without EV. On the other hand, the power-demand by the split charging schedule is about $900 \mathrm{~kW}$ lower than it by the night simultaneously charging schedule by dividing the charging time zone.

In Figs. 12-14, the line voltage of each phase by the night simultaneously charging schedule is about from
$150 \mathrm{~V}$ to $200 \mathrm{~V}$ lower compared with no EV. On the other hand, the line voltage by the split charging schedule is only about $50 \mathrm{~V}$ lower compared with no EV by dividing the charging time zone.

From these results, the night simultaneously charging by EVs and PHEVs occur the new peak of power-demand and the steep voltage drop. In addition, by dividing the charging time zone of EVs and PHEVs, it was possible to mitigate these influences.

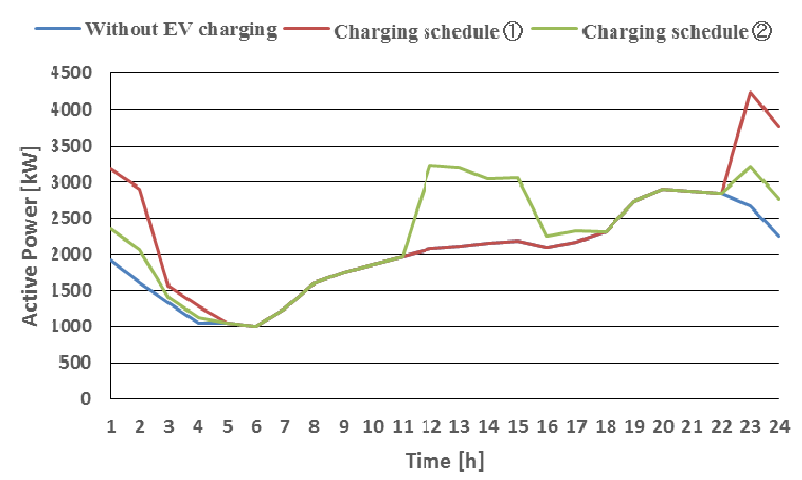

Fig. 9 Power-demand curve of residential area (heavy load period).

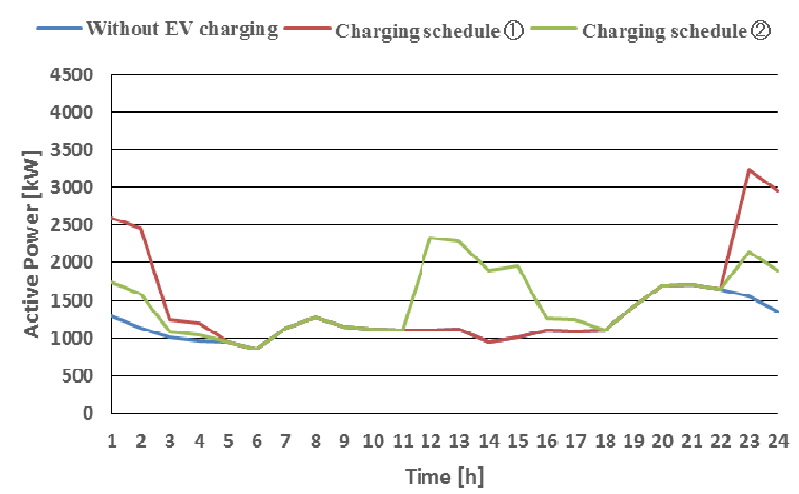

Fig. 10 Power-demand curve of residential area (light load period).

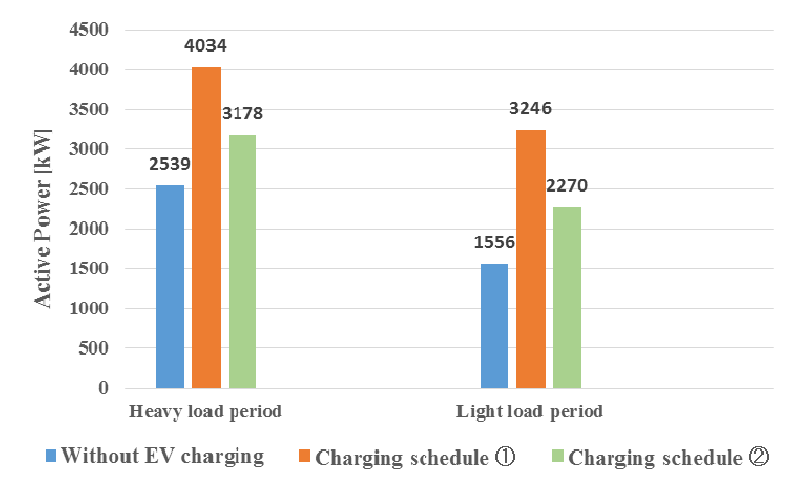

Fig. 11 Power-demand (at 11 p.m.). 


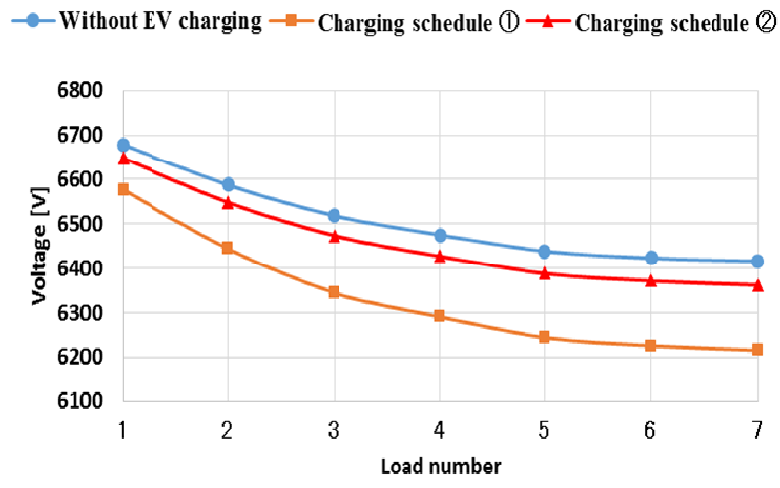

(a) Heavy load period

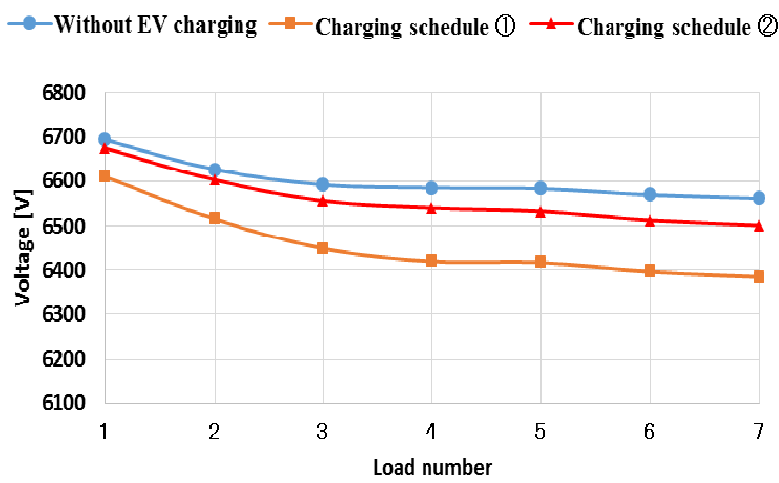

(b) Light load period

Fig. 12 Linevoltage of HV system at 11 p.m. (phase UV).

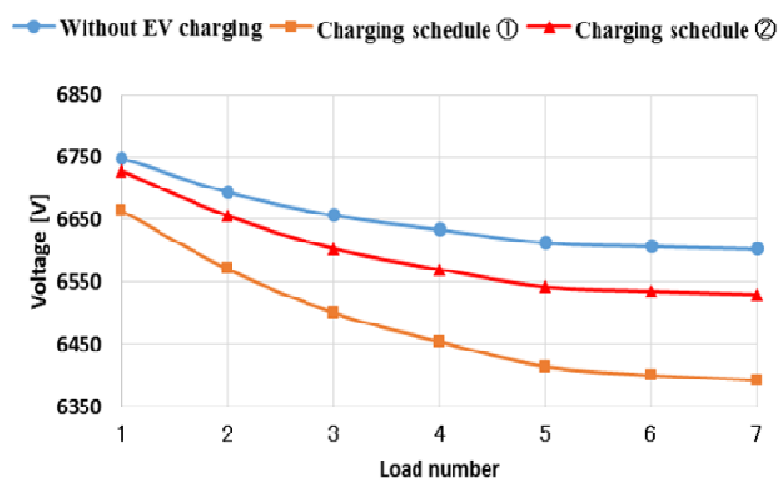

(a) Heavy load period

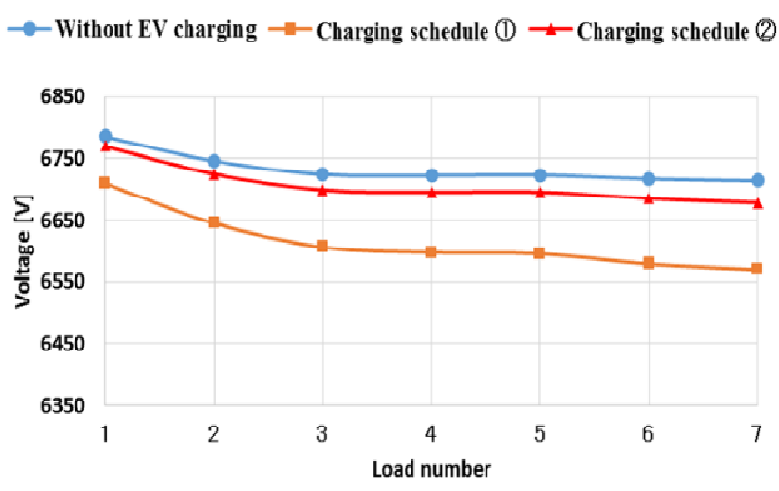

(b) Light load period

Fig. 13 Linevoltage of $\mathrm{HV}$ system at 11 p.m. (phase VW).

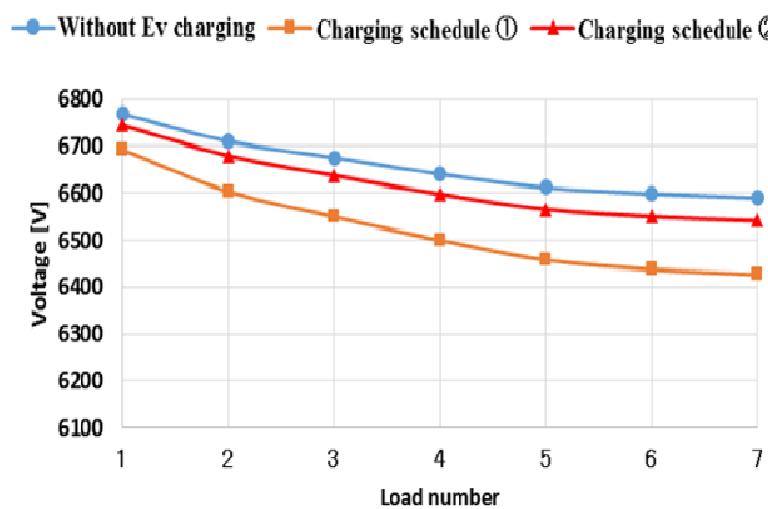

(a) Heavy load period

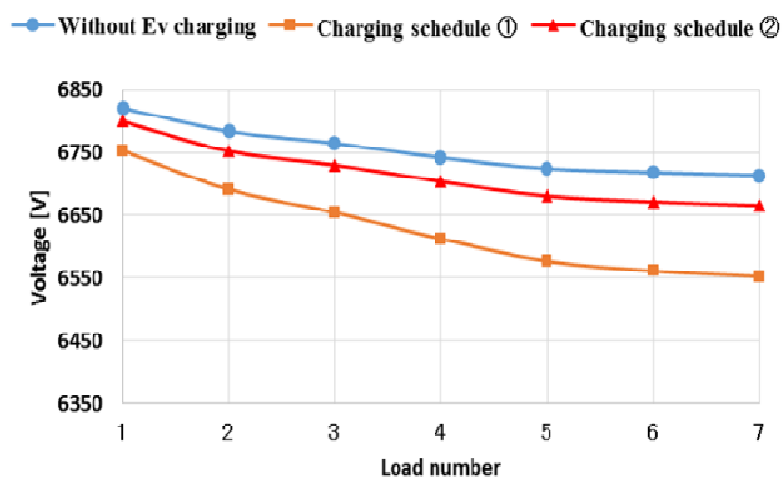

(b) Light load period

Fig. 14 Linevoltage of $\mathrm{HV}$ system at 11 p.m. (phase WU). 


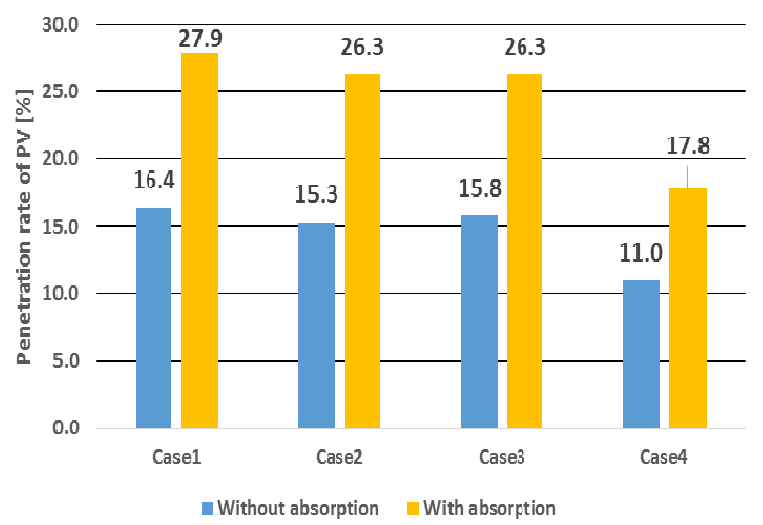

Fig. 15 Penetration rate of PVs at 12 a.m. which can avoid reverse power flow (heavy load period).

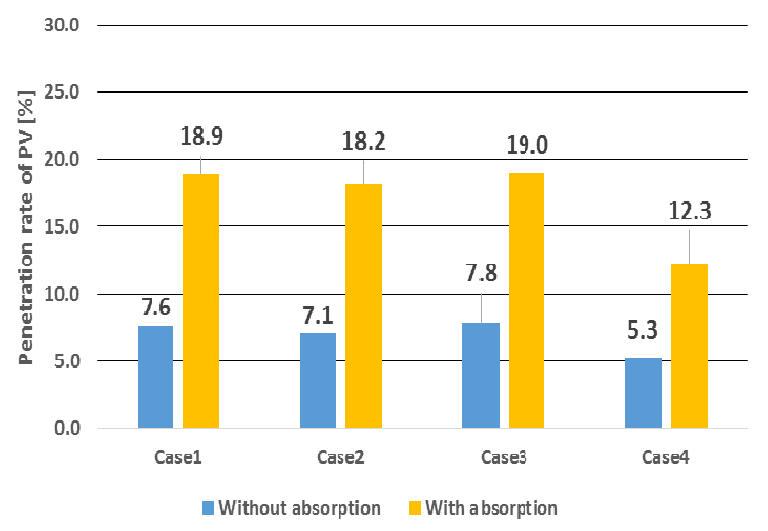

Fig. 16 Penetration rate of PVs at 12 a.m. which can avoid reverse power flow (light load period).

\section{Verification of Reverse Power Flow}

Figs. 15 and 16 show the penetration rate of PVs at 12 a.m. which can avoid the reverse power flow.

In Figs. 15 and 16, since EVs and PHEVs charged batteries at 12 a.m. according to charging schedule2, the penetration rate of PVs which can avoid the reverse power flow in each case has expanded about from $7 \%$ to $10 \%$. It is because EVs and PHEVs absorbed the surplus power produced by PVs.

\section{Conclusions}

In this study, the authors structured the distribution system model of residential area under widespread of PVs, EVs and PHEVs, and verified the influences and contributions by the two kinds of charging schedules of EVs and PHEVs. In the charging schedule 2, EVs and PHEVs based on the assumption that all EVs and PHEVs stay home at $11 \mathrm{p} . \mathrm{m}$. in case the electricity charges are low, and the charge is started from this time. In the charging schedule 2, EVs and PHEVs stay home at 12 a.m. when PV output approaches the peak, and the charge is started from this time. And EVs and PHEVs charge surplus power produced by PVs. The others start charging at 11 p.m. As a result, by the charging schedule 1 , the power-demand rose rapidly and occurred steep voltage drop at 11 p.m. On the other hand, by the charging schedule 2, the rapid power-demand rise and steep voltage drop were mitigated.

In addition, the authors changed the penetration of PVs and verified the reverse power flow produced by surplus power of PVs. In heavy load period of Case 1 under the charging schedule 1 , the reverse power flow occurred when the penetration rate exceeds $16.4 \%$. On the other hand, under the charging schedule 2, the rate expanded to $27.9 \%$. In light load period of Case 1 under the charging schedule 1 , the reverse power flow occurred when the penetration rate exceeds $7.6 \%$. On the other hand, under charging schedule 2 , the rate expanded to $18.9 \%$. And the rate expanded the penetration rate about from $7 \%$ to $10 \%$ in each case.

From the above results, when the charging time zone of electric vehicles like EVs and PHEVs is split suitably, the rapid power-demand rise and steep voltage drop are mitigated. And the charging of EVs and PHEVs can contribute to the distribution system by absorbing surplus power produced by PVs.

\section{References}

[1] Institute of Electrical Engineers of Japan Technology Report. 2008. Study on Energy Demand and Supply Management in Autonomous Demand Area Power System-Control Method and Effect by Demand Supply Interface. Report Number R07013.

[2] Koyanagi, F., and Uriu, Y. 1997. "Modeling of Electric Vehicle's Demand and Its Impact on the Daily Load." IEEJ Trans. PE, IEEJ 117 (1): 41-6.

[3] Next-Generation Vehicle Strategy. 2010. Japanese Ministry of Economy, Trade and Industry. 
http://www.meti.go.jp/committee/summary/0004630/pdf/ 20100412002-3.pdf.

[4] FUJI KEIZAI CO., LTD, HEV, EV-Related Market thorough Analysis 2013. http://www.group.fuji-keizai.co.jp/press/pdf/130301_130 18.pdf.

[5] NHK Broadcasting Culture Research Institute Report. 2010. Survey of How the Nation's People Use Their Time. https://www.nhk.or.jp/bunken/summary/yoron/lifetime/p df/110223.pdf.

[6] Ministry of Land, Infrastructure, Transport and Tourism. 2012. National Survey of City Traffic Characteristics. http://www.mlit.go.jp/common/001032141.pdf.

[7] Implemental Report of the Demonstration Experiment of EVs and PHVs in Saitama Prefecture. 2013. https://www.pref.saitama.lg.jp/a0502/documents/613045. 